\title{
Transient AV Complete Heart Block: A Rare Complication Following Regadenoson Injection
}

\author{
Umut Gomceli $^{1 *}$, Manoj Bhandari², Jonathan N Bella² ${ }^{2}$ and Gayathri Kamalakkannan ${ }^{2}$ \\ ${ }^{1}$ Division of Cardiology, Mount Sinai Saint Luke's Hospital, USA \\ ${ }^{2}$ Division of Cardiology, Bronx Care Health System, US
}

*Corresponding author: Umut Gomceli, Division of Cardiology, Mount Sinai Saint Luke's Hospital-Mount Sinai West-Bronx Care Health System, USA.

To Cite This Article: Umut Gomceli, Transient AV Complete Heart Block: A Rare Complication Following Regadenoson Injection. Am J Biomed Sci \& Res. 2020 - 7(2). AJBSR.MS.ID.001127. DOI: 10.34297/AJBSR.2020.07.001127.

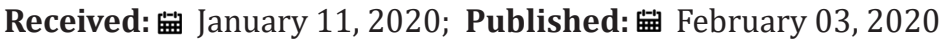

\begin{abstract}
Regadenoson is an $\mathrm{A} 2 \mathrm{~A}$ adenosine receptor agonist designed to selectively and transiently produce coronary vasodilation. Although regadenoson is a relatively selective A2A receptor agonist, it also has some weak action on the A1 receptor (10-fold lower affinity) which can produce atrioventricular block and bradycardia [1]. Regadenoson was approved by the FDA in 2008 for use as a pharmacologic stress agent during radionuclide myocardial perfusion imaging [2]. Well-documented contraindications for regadenoson use are second- or third-degree AV block or sinus node dysfunction without a functioning pacemaker (ASNC guideline reference). As of June 2017, a total of 56 cases of third-degree heart block and 26 cases of sinus arrest associated with regadenoson stress testing were reported via FAERS [3]. The incidence of overall high-grade AV block defined as second and third-degree AV block related to the administration of regadenoson at the dose given during MPI was low (less than 0.5\%) and observed much less frequent compared to adenosine [4]. We report a case of 49-year-old woman who was referred to our stress laboratory for nuclear stress imaging with regadenoson and developed atrioventricular complete heart block (AVCHB) after regadenosone injection.
\end{abstract}

\section{Case Report}

A 49-year-old woman with hypertension, diabetes mellitus type II, chronic Kidney disease stage III, hyperlipidemia, asthma, moderate obstructive sleep apnea, neuropathy and recent cystourethroscopy and stent placement. A transthoracic echocardiogram was obtained as a part of pre-operative evaluation for decreased exercise tolerance showed decreased Left ventricle ejection fraction (LVEF). She was referred for cardiology evaluation post-surgery for newly diagnosed cardiomyopathy. She reported atypical chest pain with limited exercise capacity. She was referred for pharmacological nuclear stress test, given multiple coronary artery disease risk factors and decreased LVEF. Pre-test electrocardiogram showed normal sinus rhythm with heart rate of 86 bpm, normal axis without ST/T segment changes and normal QT segment. Resting blood pressure was 156/85 mmHg. Pre-stress physical exam was unremarkable.

Figure 1: Resting ECG in normal sinus rhythm. Heart rate at $86 \mathrm{bpm}$. 


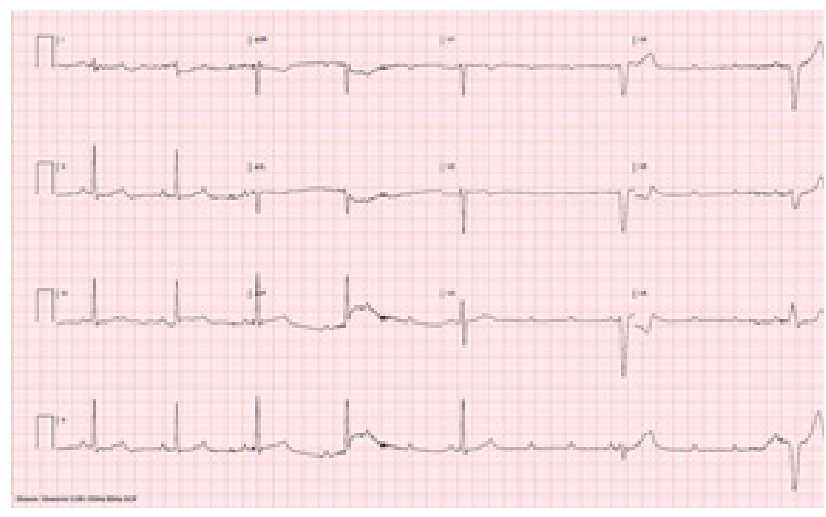

Figure 2: Initial Development of 2:1 AV block and subsequent progression AV complete Heart block and Ventricular escape rhythm following 40 second. of regadenoson injection and lasted for 20 seconds.

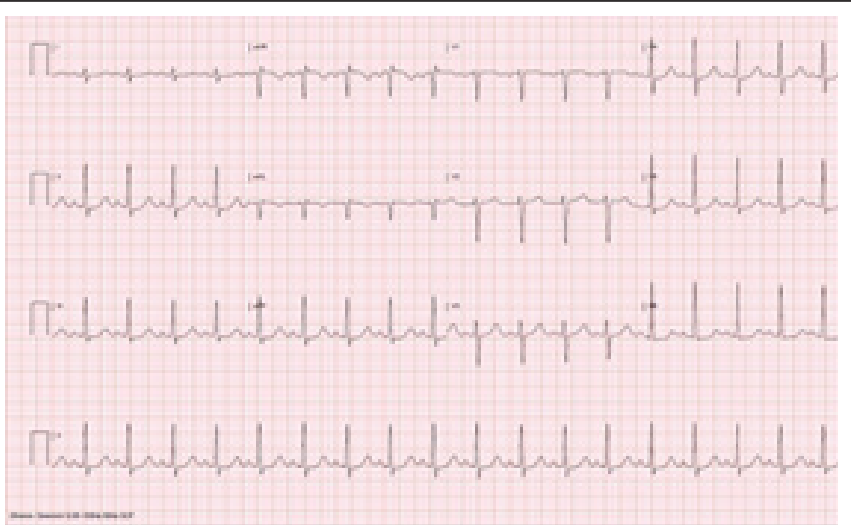

Figure 3: Immediate recovery of the conduction as sinus tachycardia following intravenous $75 \mathrm{mg}$ aminophylline injection.

Following intravenous injection of regadenoson $(0.4 \mathrm{mg})$ patient developed sinus bradycardia which progressed to $\mathrm{AV}$ complete heart block for 20 seconds. Normal AV conduction was recovered as sinus tachycardia following immediate intravenous $75 \mathrm{mg}$ aminophylline injection. Patient did not lose consciousness however felt extremely nauseated and subsequently vomited a few times. Patient was observed in the stress laboratory for 30 minutes, she remained hemodynamically stable and there was no recurrence of $\mathrm{AV}$ block. Patient completed Myocardial Perfusion Imaging (MPI) scan which showed moderate size, moderate intensity partially reversible defect extending from the apex to the base of the anterior wall on SPECT images, mildly enlarged LV size and moderately decreased systolic function on gated images. Further evaluation with diagnostic catheterization as outpatient showed non-obstructive coronary artery disease (Figures 1-3).

\section{Discussion}

Regadenoson, is easier to use and associated with better safety and tolerability profiles than non-selective agents such as adenosine and dipyridamole. Vast majority of these side effects are short-lived, benign, and spontaneously terminate. On rare occasions, however, more serious cardiovascular and neurological adverse events may develop, namely symptomatic myocardial ischemia, infarction, high-grade AV block, asystole, and seizures [5].
Although its affinity at the A1 receptor has been reported to be 10-15-fold weaker, this remains important as these receptors are found at the sinoatrial and atrioventricular node in addition to atrial and ventricular myocytes [6] which is likely the potential source of bradyarrhythmic complications. Also, there has been a theoretical concern of increased and prolonged side effects in patients with chronic kidney disease; however, prior work has demonstrated safety and tolerability in end stage renal disease patients $[7,8]$. Among those totals of 7 reported high degree AV block patients only 2 reportedly had chronic kidney failure. Furthermore, clinical information regarding the 47 cases of complete heart block and 25 cases of sinus arrest reported via the FDA adverse event reporting system (FAERS) is not available [9] hence the real prevalence of regadenoson-induced high-degree AV block remains unknown. It is difficult to predict if a patient with a normal ECG will develop a complete heart block following regadenoson injection. It is important to stress the importance of recognizing potential side effects and treating them immediately [10]. In our case immediate reversal with aminophylline restored sinus rhythm with normal conduction and improvement in symptoms.

We believe the awareness of this potential side effect in nuclear laboratories and readiness of the equipment and drugs in emergency situations is important. 


\section{Disclosure}

Authors have no disclosure.

\section{References}

1. Prenner BM, Bukofzer S, Behm S, Feaheny K, McNutt BE (2012) A randomized, double-blind, placebo-controlled study assessing the safety and tolerability of regadenoson in subjects with asthma or chronic obstructive pulmonary disease. J Nucl Cardiol 19(4): 681-692.

2. Al Jaroudi W, Iskandrian AE Regadenoson (2009) A new myocardial stress agent. J Am Coll Cardiol 54(13): 1123-1130.

3. Andrikopoulou E, Morgan CJ, Brice L (2017) Incidence of atrioventricular block with vasodilator stress spect, A meta-analysis. J Nucl Cardiol 26(2): 616-628.

4. Andrikopoulou E, Hage FG (2018) Adverse effects associated with regadenoson myocardial perfusion imaging. Journal of nuclear cardiology: official publication of the American Society of Nuclear Cardiology 25(5): 1724-1731.

5. Zoghbi GJ, Iskandrian AE (2012) Selective adenosine agonists and myocardial perfusion imaging. Journal of nuclear cardiology 19(1): 126141.
6. Doukky R, Rangel MO, Wassouf M, Dick R, Alqaid A, et al. (2013) The safety and tolerability of regadenoson in patients with end-stage renal disease: The first prospective evaluation. Journal of nuclear cardiology official publication of the American Society of Nuclear Cardiology 20(2): 205-213.

7. Gordi T, Blackburn B, Lieu H Regadenoson (2007) pharmacokinetics and tolerability in subjects with impaired renal function. Journal of clinical pharmacology 47(7): 825-833.

8. Kureshi F, Abdallah MS, Bateman TM (2017) Regadenoson-induced complete heart block and asystole: A real possibility nuclear laboratories should be aware of Journal of nuclear cardiology 24(6): 2019-2024.

9. Pandit A, Unzek Freiman S (2012) Complete heart block associated with regadenoson. A real side effect. Journal of nuclear cardiology 9(6): 12361239. 